\title{
Towards incorporating affective computing to virtual rehabilitation; surrogating attributed attention from posture for boosting therapy adaptation
}

\author{
Jesús J. Rivas ${ }^{a}$, Patrick Heyer ${ }^{a}$, Felipe Orihuela-Espina ${ }^{a}$ and Luis Enrique Sucar ${ }^{a}$ \\ ${ }^{a}$ Instituto Nacional de Astrofísica, Óptica y Electrónica (INAOE), Sta. Ma. Tonantzintla, \\ Puebla, Mexico;
}

\begin{abstract}
Virtual rehabilitation (VR) is a novel motor rehabilitation therapy in which the rehabilitation exercises occurs through interaction with bespoken virtual environments. These virtual environments dynamically adapt their activity to match the therapy progress. Adaptation should be guided by the cognitive and emotional state of the patient, none of which are directly observable. Here, we present our first steps towards inferring non-observable attentional state from unobtrusively observable seated posture, so that this knowledge can later be exploited by a VR platform to modulate its behaviour. The space of seated postures was discretized and 648 pictures of acted representations were exposed to crowd-evaluation to determine attributed state of attention. A semi-supervised classifier based on Naïve Bayes with structural improvement was learnt to unfold a predictive relation between posture and attributed attention. Internal validity was established following a $2 \times 5$ cross-fold strategy. Following 4959 votes from crowd, classification accuracy reached a promissory 96.29\% $(\mu \pm \sigma=87.59 \pm 6.59)$ and F-measure reached $82.35 \%(\mu \pm \sigma=69.72 \pm 10.50)$. With the afforded rate of classification, we believe it is safe to claim posture as a reliable proxy for attributed attentional state. It follows that unobtrusively monitoring posture can be exploited for guiding an intelligent adaptation in a virtual rehabilitation platform. This study further helps to identify critical aspects of posture permitting inference of attention.
\end{abstract}

Keywords: Attention, Adaptation, Posture, Neurorehabilitation, Semi-supervised learning

\section{INTRODUCTION}

Several medical conditions such as stroke, palsy, dystonia, or traumatic brain injury can lead to motor impairment. To recover motor dexterity and functional ability, the affected people follow expensive, long and demotivating rehabilitation treatments often resulting in abandonment of the therapy. Virtual rehabilitation $(\mathrm{VR})^{1}$ is a novel motor rehabilitation therapy exploiting virtual reality to present the rehabilitation exercises to the impaired patient in safe and fully customizable training environments. It promises to reduce therapy costs, to facilitate treatment at home and to enhance motivation to increase adherence to therapy. ${ }^{2}$

Dynamic customization, also referred to as adaptation, is the ability of the VR to evolve its behaviour concomitantly with the changing needs of the patient. ${ }^{3}$ It is arguably the key element behind the VR capacity to fulfill intelligent personalization conciliating the patient ongoing necessities with the therapist's long term plan, and it is realized by means of artificial intelligence (AI) solutions. Most AI strategies for adaptation in VR so far have been naïve and based on observable behaviour, e.g. speed and control. ${ }^{3}$ Recently, they are starting to further tapping the non-observable cognitive and emotional state of the patient. ${ }^{4}$ It is expectable that supplementing adaptation strategies with cognitive and emotional input can (i) foster motivation, and (ii) optimize exploitation of learning-related plastic changes in the brain.

Affective computing is a branch of human-computer interaction dedicated to the design of systems that can recognize, interpret, and simulate human emotions and related affective phenomena*. Affective computing

Further author information: (Send correspondence to L.E.S.)

J.J.R.: E-mail: jjoelrivas@gmail.com, Telephone: +52 (222) 2663100

L.E.S.: E-mail: esucar@ccc.inaoep.mx, Telephone: +52 (222) 2663100 Ext. 8208

*IEEE Transactions on Affective Computing. Aims and scope. http://www.computer.org/portal/web/tac. 3-May2014

10th International Symposium on Medical Information Processing and Analysis,

edited by Eduardo Romero, Natasha Lepore, Proc. of SPIE Vol. 9287, 92870Y

(c) 2015 SPIE $\cdot$ CCC code: 1605-7422/15/\$18 · doi: 10.1117/12.2072426

Proc. of SPIE Vol. $928792870 Y-1$ 
is therefore a forefront candidate computational paradigm to interrogate the mind (cognitive and emotional) state of the patient. Of the many processes concurrently conforming this mind state, this paper focuses on attention. Attention is the capacity to opt out one or several stimuli among potential distracters. ${ }^{5}$ Monitoring the rehabilitation patient's attention while interacting with the virtual environment requires either (a) obtrusively recording neurological activity by means of neuroimaging tools e.g. electroencephalography (EEG) or (b) find a physiological or behavioural surrogate that can be monitored unobtrusively and can reliably encode attention. Physiological information afford a more direct measurement of attention, however, almost unavoidably will require contact sensing. Alternatively, behavioural surrogates can only provide an indirect measurement of attention, that is attributed attention, but can be achieved contactless by video monitoring.

Static body postures can be mined for regulators communicating the attentional and affective state of subjects during normal human communication process. ${ }^{6}$ Hence, we hypothesize that posture can act as a behavioural proxy of attributed attention. Moreover, body posture can be continuously silently watched in real time with traditional video cameras, and more recently with sensors like the Kinect. Considering the previous work at our lab to develop a VR platform for the upper limb, ${ }^{2}$ we are particularly interested in seated postures as training for the upper limb often occurs with the patient seated in front of the VR platform.

Consequently, in this paper, we present our early work towards integrating elements of affective computing to the field of virtual rehabilitation with the aim of enriching the adaptation policy. For this, we present an initial experiment aimed at elucidating whether seated posture can reliably surrogate attributed attention. Shall this predictive relation (not necessarily explanatory) be confirmed by high classification rates, it is conceivable that monitoring of posture can be used to augment therapy adaptation to the impaired patient.

\section{RELATED WORK}

\subsection{Attentional models}

Attention is not a unitary construct or mechanism but rather a characteristic pervading multiple perceptual and cognitive control mechanisms. ${ }^{5}$ Different attentional models co-exist addressing different dimensions of the attentional construct e.g. the target of the attention whether external or internal, ${ }^{5}$ the mechanism of attention whether exogenous or bottom-up (stimulus driven) versus endogenous or top-down (goal driven) ${ }^{7,8}$ or whether attention is overt (accompanied by eye shift) or covert (restricted to mental shift) ${ }^{9}$ among others. These miscellaneous dimensions are processed by various brain networks. At least three distinct but related brain networks, ${ }^{10,11}$ but likely more ${ }^{12}$ underlie attentional processes. This three-network approach is limited ${ }^{12}$ because, for the sake of experimental feasibility and control, it privileges attention as a mainly perceptuallyrelated cognitive process. As such it ignores that, in real-life contexts people rely on attention to guide bodily action. $^{13}$

\subsection{Attention from posture}

A host of psychophysiological measures have begun to yield robust indexes related to the instantaneous attentional state of a subject, ${ }^{14-16}$ his or her emotional disposition towards the task at hand, ${ }^{17,18}$ and whether he or she is in an exploratory attitude or a more self-involved reflective moment. ${ }^{19,20}$ In parallel, behavioural measures that can be assessed contactless are also being started to be mined as proxies of emotions. In this sense, the observable conduct as characterized by the body gestures and sitting postures conveys information related to affective states, ${ }^{21,22}$ and in particular about attributed attention. ${ }^{22}$ Previous work has coarsely regarded the participant's upper body in 6 main postures: ${ }^{23}$ upright, leaning back, leaning forward, sitting at the front edge, leaning left/right, slouching. These can conform a basic lexicon of postures to which postural analysis may be referred back to. In addtion, it is known that the head supplies more information about the nature of an emotion, while the body provides more information about the intensity of an emotion. ${ }^{24}$

With these premises, seated postural analysis in human-computer interfaces has already been attempted by means of varied sensing technologies and with the aim to investigate a range of emotions. ${ }^{22,25-28}$ Without entering into details, state of the art in decoding different emotional states in specific contextual tasks is in the range of high 70s to low 80s percentages of accuracy depending on the targeted emotion. It is yet unclear whether these achievements are based on a proper understanding of the behavioural expression i.e. robust features leading to 


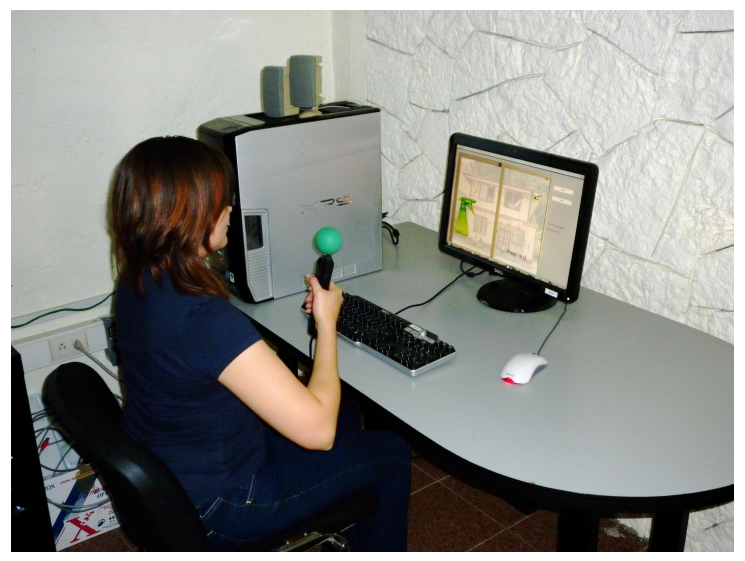

Figure 1. A user exercising in the VR platform Gesture Therapy developed by our group. Interaction with the virtual environments occurs by tracking the colour ball on top of the gripper controller representing arm displacement by means of computer vision algorithms, as well as direct sensing of pressure indicative of flexion and extension of fingers with a sensor on the front of the gripper.

good classification rates irrespective of the analysis approach, or the consequence of a successful ad-hoc analysis. The former is more likely to be generalizable.

\section{VIRTUAL REHABILITATION AND THE GESTURE THERAPY}

Any neurorehabilitation therapy, whether compensatory or focusing on impairment, ${ }^{29}$ should exploit the principles of experience-dependent neural plasticity ${ }^{30}$ to be sucessful. $\mathrm{VR}^{31}$ departs from a priviledged position to fulfill these principles and do so in a personalized manner considering its capacity to present therapy stimulus within adaptable training environments. Our group has developed a particular instantiation of the VR paradigm, called Gesture Therapy $(\mathrm{GT})^{2,32}$ illustrated in Figure 1. Similarly to other VR solutions, GT presents the rehabilitatory exercises disguised as actions that the user has repetitively execute to complete certain tasks within the virtual environments incorporated to the platform. One of the distinguishing features of GT when compared to other alternatives is its strong AI model guiding the adaptation process. This model combines (i) an a priori optimal initialization of the adaptation policy achieved with a Markov decision process with (ii) a dynamic updating of the policy as new observations of the user performance and feedback from the therapist becomes available realised with reinforcement learning. ${ }^{3}$ Input to this model, however, only exploits metrics of performance such as speed and control limiting its ability to adapt challenge to match the internal cognitive state of the patient.

\section{METHODS}

\subsection{Crowd-sourcing: Building a ground truth of attributed attention}

The space of seated postures was discretized in terms of 6 degrees of freedom summarised in Table 1 yielding 216 possible postures. These were modelled in a $3 \mathrm{D}$ virtual mannequin. The synthetic model was presented to 4 young volunteer human models of both genders that acted the posture whilst a picture and the corresponding skeleton of the posture were captured with a Kinect from a frontal view. Picture acquisition was carried out at the National Institute of Astrophysics, Optics and Electronics (INAOE) in Mexico. Fig. 2 shows an example of the virtual mannequin and the human model acting the indicated posture. To ensure that only posture will guide attribution of attention by human raters some factors were controlled as illustrated in Fig. 2; the face of the model was covered by a inexpressive mask, the model was dressed with a grey jumper, when appropriate the hair was combed tied in a ponytail and the photograph session was carried out in a room with minimal furniture and a uniform pastel shaded wall.

To limit fatigue of the human models, picture acquisition was split in two sessions (different dates) of 108 postures each time presented in random order. Two of the human models completed the full collection, and the 
Table 1. Degrees of freedom used for the discretization of the space of seating postures.

\begin{tabular}{|c|l|l|}
\hline Acronym & Description & Levels \\
\hline Tor & Inclination of the torso in degrees & $0:$ sitting straight, 30: tilted forward \\
\hline Sho & Inclination of the shoulders & $-10:$ hunched back, 0: normal straight, 10: driven forth. \\
\hline Rar & Tilt of the right arm in degrees & $0:$ Aligned to the vertical axis, 45: bent upward \\
\hline Lar & Tilt of the left arm in degrees & $0:$ Aligned to the vertical axis, 45: bent upward \\
\hline HeH & Horizontal tilt of the head in degrees & $-30:$ tilted left, 0: straight, 30: tilted right \\
\hline HeV & Vertical tilt of the head in degrees & $-30:$ tilted left, 0: straight, 30: tilted right \\
\hline
\end{tabular}
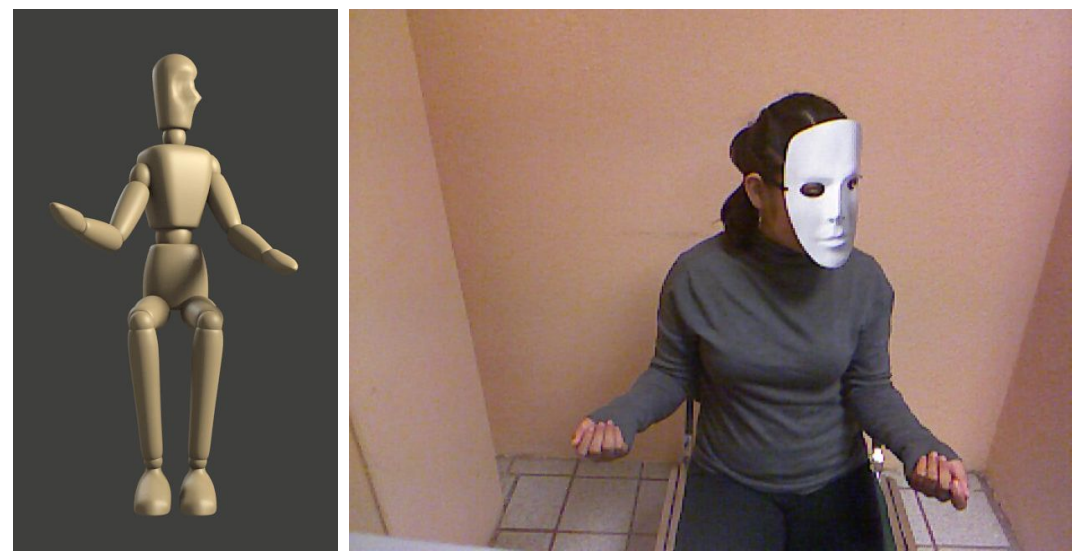

Figure 2. Left: The illustrative 3D virtual mannequin exemplifying the posture for the model. Right: The human model enacting the posture that will be part of the repository of seated postures.

other two models only half. In total, a repository of 648 images of acted seated postures were obtained with a minimum of 3 photos per posture guaranteeing at least a minimal intra-postural variability. This repository was made available to a large number of independent raters blind to each other. Crowd-sourcing is a recent paradigm encapsulating the idea of obtaining services from a large group of people, often through the Internet. The set of pictures was uploaded into a website hosted in our institution servers ${ }^{\dagger}$ specifically set up for collecting crowd votes. Open invitations for voting were sent out by e-mail, both internally and externally to the institution. Each volunteer rater would sign in with his/her e-mail and vote as many of the pictures as he wants until he voluntarily stops, and he may continue at any other time. No attempt was made to control for multiple aliases. Pictures are presented to the raters in a randomised order but ensuring that the same rater do not see the same picture more than once. For each picture, the web interface provides two voting options "Attentive" (AT) and "Not attentive" (NA). Intentionally, if raters make a mistake, they have no chance of correcting their choice; this introduces error e.g. variability, in the ratings, which might alleviate possible issues of overfitting during the building of the classifier. Collaterally, this accelerates the voting process.

\subsection{Classification model}

A naïve Bayes ${ }^{33}$ with structural improvement classifier model was learnt from the postures labeled dataset. Structural improvement is achieved by means of eliminating those uninformative features and grouping those highly redundant as established with mutual information.

The naive or simple Bayesian classifier (NBC) is based on the assumption that all the attributes are independent given the class variable; that is, each attribute $A_{i}$ is conditionally independent of all the other attributes given the class: $P\left(A_{i} \mid A_{j}, C\right)=P\left(A_{i} \mid C\right), \forall j \neq i$. Under this assumption, the posterior probability of the class

\footnotetext{
${ }^{\dagger}$ http://robotic.inaoep.mx/postura/
} 
$C$ given the attributes $A_{1}, \ldots A_{n}$ is:

$$
P\left(C \mid A_{1}, A_{2}, \ldots, A_{n}\right)=P(C) P\left(A_{1} \mid C\right) P\left(A_{2} \mid C\right) \ldots P\left(A_{n} \mid C\right) / P(\mathbf{A})
$$

The performance of the naive Bayes classifier can be improved by a structural improvement: ${ }^{34}$ eliminating irrelevant attributes and eliminating or joining dependent attributes. This has as an advantage that the efficiency and simplicity of the NBC is maintained, and at the same time the performance is improved for cases where the attributes are not independent. This type of Bayesian classifiers are known as Semi-Naive Bayesian Classifiers (SNBC).

Node elimination consists on simply eliminating an attribute, $A_{i}$, from the model, this could be because it is not relevant for the class $\left(A_{i}\right.$ and $C$ are independent); or because the attribute $A_{i}$ and another attribute, $A_{j}$, are not independent given the class. Node combination consists in merging two attributes, $A_{i}$ and $A_{j}$, into a new attribute $A_{k}$, such that $A_{k}$ has as possible values the cross product of the values of $A_{i}$ and $A_{j}$ (assuming discrete attributes). For example, if $A_{i}=a, b, c$ and $A_{j}=1,2$, then $A_{k}=a 1, a 2, b 1, b 2, c 1, c 2$. This is an alternative when two attributes are not independent given the class. By merging them into a single combined attribute, the independence condition is not longer relevant.

Which attributes to eliminate or combined is based on measuring the mutual information (MI) between the class and each attribute $A_{i}$ (eliminate $A_{i}$ if MI is low); and measuring the conditional mutual information (CMI) between $A_{i}$ and $A_{j}$ given the class (eliminate $A_{i}$ or $A_{j}$ or combine them if CMI is high). In the second case, the best alternative is selected by cross validation.

The initial focus of the experimental exercise was two-fold; (i) to establish a predictive relation, and (ii) to identify the specific degrees of freedom guiding this relation. To achieve this, other computational approaches are certainly feasible, and moreover improving of classification rates is likely to occur under more aggressive strategies such as full model selection ${ }^{35}$ and/or deep learning. ${ }^{36}$ But these engineer ad-hoc representations boosting the first goal but compromising the second goal; and we favour revealing robust factors of variation transcendant of the analytical strategy which is more likely to occur with a well understood white-box classifier such as naïve Bayes.

Internal validity of the classifier model was established using the cross validation mechanism with 2 iterations of $k=5$ folds i.e. $2 \times 5 \mathrm{cv}$.

Individual pictures were coded with the 6 dimensional feature vector encoding the underlying posture with each of the six aforementioned degrees of freedom respectively (see Table 1). Each picture is considered a case or observation for the machine learning process. They were further assigned attentional class, AT or NA, following a simple majority voting scheme. However, to make the most of our crowd-evaluation, a semi-supervised learning approach was used to build our classification model. In semi-supervised learning, the model is learnt iteratively using first, those cases for which the class is known with higher confidence, and then in later iterations new cases with progressively less confidence are added, and confidence levels updated. In order to establish the initial confidence on the class assigned to each posture case, votes were aggregated for each posture. The absolute difference in votes between classes (AT and NA) was calculated for each posture and this difference was used a measure of confidence in class label. Pictures may initially have no votes (due to the random presentation of the associated picture) -class unknown-, an equal number of votes favouring each attentional state -total lack of confidence in class label-, or a certain difference of votes between classes -the higher the difference the higher the confidence in class label-.

\section{RESULTS}

\subsection{Crowd-sourcing evaluation}

After an arbitrary period of around two months in which votes were collected, voting data was retrieved from the server. In total, 4959 votes were obtained. The votes of all raters were given equal weight. The website remains accessible and open to further voting for future usage, but data collected since is not further considered in this paper. Votes are distributed asymmetrically as illustrated in Fig. 3, leading to unbalanced classification. However, no effort has been made to correct for this degeneracy. 


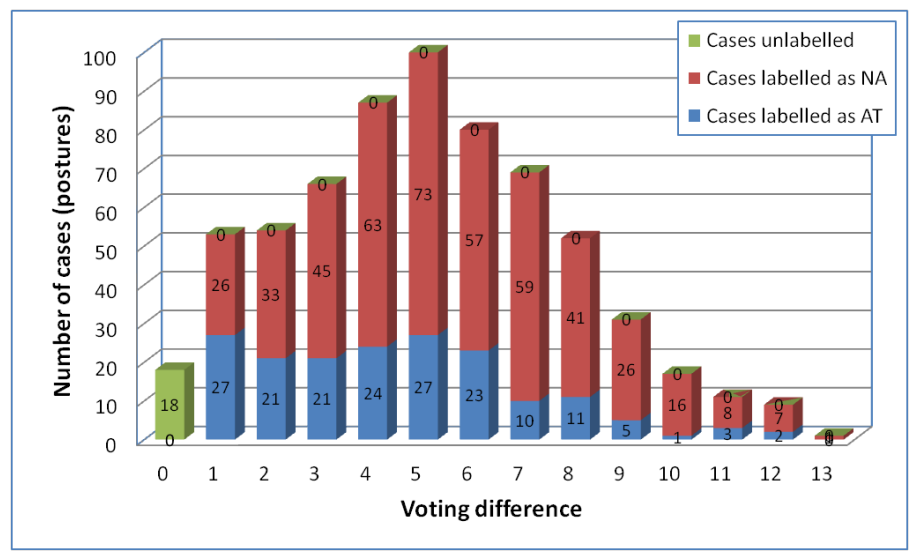

Figure 3. The distribution of the picture labelling voting according to class labelling confidence.

\subsection{Classification results}

Following observation of the voting distribution, an arbitrary threshold was set at 6 for choosing the initial training set for the semi-supervised learning, yielding 270 labeled examples considered to be correctly classified at training start. Across the $2 \times 5 \mathrm{cv}$, the best model reached a maximum classification accuracy $\ddagger$ of $90.74 \%$ (mean \pm std: $81.48 \pm 7.85)$ and F-measure reached $82.35 \%(\mu \pm \sigma=69.72 \pm 10.50)$. The latter represented a significant improvement over the basic classifiers without structural improvement (Mann-Whitney-U test: $p<.05$ ). We are however severely affected by the class unbalance which manisfest as high specificity but low sensitivity (see Table 2).

Table 2. Summary of internal validity using $2 \times 5$ cross-fold mechanism. Results are indicated as mean \pm std.

\begin{tabular}{|l|l|c|c|c|c|c|}
\hline Stage & Set & Accuracy & Sensitivity (Recall) & Specificity & Precision & F-Measure \\
\hline Naive Bayes only & Train & $85.0 \pm 2.64$ & $46.60 \pm 18.75$ & $94.77 \pm 2.01$ & $69.31 \pm 7.40$ & $54.07 \pm 15.72$ \\
\hline Naive Bayes only & Test & $81.84 \pm 7.85$ & $37.37 \pm 22.64$ & $92.80 \pm 3.16$ & $57.86 \pm 24.02$ & $43.01 \pm 21.15$ \\
\hline with Struct. Impr. & Train & $84.53 \pm 2.48$ & $50.63 \pm 20.32$ & $93.19 \pm 2.71$ & $65.93 \pm 4.79$ & $60.45 \pm 7.90$ \\
\hline with Struct. Impr. & Test & $87.59 \pm 6.59$ & $56.37 \pm 22.32$ & $95.87 \pm 3.70$ & $80.46 \pm 15.43$ & $69.72 \pm 10.50$ \\
\hline
\end{tabular}

Based on the mutual information associated to each postural feature, $\mathrm{HeH}$ and $\mathrm{HeV}$, i.e. the horizontal and vertical positions of the head were found to be the postural features that most contribute to the determination of the attributed attentional state. Following structural improvement, the Rar and Tor postural feature were discarded, and the Lar and $\mathrm{HeV}$ attributes were joint.

\section{CONCLUSIONS}

Our result suggest that it is possible to establish a predictive relationship e.g. high accuracy, between constraint acted seated posture and crowd-attributed attention using an structurally enhanced naïve Bayes classifier and benefiting from the semi-supervised learning scheme, although further work is necessary to improve sensitivity. Hence, it seems reasonable to envisage the integration of attentional state of the patient to the adaptation policy of a VR platform using unobtrusive sensing. Limitations and biases of this study include coarse discretization of the postural space, low intra-postural variability and low content validity due to the cohort of human models and binarization of the attentional state, predefined analysis strategy i.e. no other classifiers have been tested and

\footnotetext{
${ }^{\ddagger} \mathrm{TP}$ : true positive, $\mathrm{TN}$ : true negative, $\mathrm{FP}$ : false positive, $\mathrm{FN}$ : false negative; accuracy $=(\mathrm{TP}+\mathrm{TN}) /(\mathrm{TP}+\mathrm{TN}+\mathrm{FP}+\mathrm{FN})$; sensitivity or recall $=\mathrm{TP} /(\mathrm{TP}+\mathrm{FN}) ; \quad$ specificity $=\mathrm{TN} /(\mathrm{TN}+\mathrm{FP}) ; \quad \operatorname{precision}=\mathrm{TP} /(\mathrm{TP}+\mathrm{FP}) ; \quad \mathrm{F}-$ measure $=2($ precision $*$ recall $) /($ precision + recall $)$.
} 
constraints imposed to contextual usage of the scenario as well as cultural homogeneity of the models. Our next steps shall be improving sensitivity rates and to exploit this affective computing knowledge to enrich the current adaptation capabilities of Gesture Therapy, as well as exploring other affective elements such as frustration, not necessarily sensed from posture, as possible candidates to further augment adaptation to the patient.

\section{ACKNOWLEDGMENTS}

We thank Dr. Diego Cosmelli for his valuable communications regarding attentional models. Research funded by Microsoft LACCIR research project R1211LAC0001 and project SIREEX from the Mexican CONACYT 218709.

\section{REFERENCES}

[1] Levin, M. F., "Can virtual reality offer enriched environments for rehabilitation?," Expert Reviews of Neurotherapeutics 11(2), 153-155 (2011).

[2] Sucar, L. E., Orihuela-Espina, F., Velázquez, R. L., Reinkensmeyer, D. J., Leder, R., and HernándezFranco, J., "Gesture therapy: An upper limb virtual reality-based motor rehabilitation platform," IEEE Transactions on Neural Systems and Rehabilitation Engineering 22(3), 634-643 (2014).

[3] Ávila-Sansores, S. M., Orihuela-Espina, F., Sucar, L. E., and Álvarez-Cárdenas, P., "Adaptive decision models for virtual rehabilitation environments," in [International Conference on Machine Learning (ICML'2013) Workshop on Role of Machine Learning in Transforming Healthcare (WHEALTH)], (16-21st JUN 2013).

[4] Sucar, L. E., Ávila-Sansores, S. M., and Orihuela-Espina, F., [Submitted to Foundations of Biomedical Knowledge Representation], ch. User Modelling for Patient Tailored Virtual Rehabilitation, 28 pp. (2014).

[5] Chun, M. M., Golomb, J. D., and Turk-Browne, N. B., "A taxonomy of external and internal attention," Annual Review of Psychology 62, 73-101 (2011).

[6] Ekman, P. and Friesen, W. V., "The repertoire of nonverbal behavior: categories, origins, usage, and coding," Semiotica 1, 49-98 (1969).

[7] Itti, L. and Koch, C., "Computational modelling of visual attention," Nature Reviews Neuroscience 2, 1-11 (2001).

[8] Treisman, A. M. and Gelade, G., "A feature-integration theory of attention," Cognitive Psychology 12, 97-136 (1980).

[9] Hunt, A. R. and Kingstone, A., "Covert and overt voluntary attention: linked or independent?," Cognitive Brain Research 18, 102-105 (2003).

[10] Posner, M. I. and Rothbart, M. K., "Research on attention networks as a model for the integration of psychological science," Annual Review of Psychology 58, 1-23 (2007).

[11] Raz, A. and Buhle, J., "Typologies of attentional networks," Nature Reviews Neuroscience 7, 367-379 (2007).

[12] Baldauf, D. and Deubel, H., "Attentional landscapes in reaching and grasping," Vision Research 50(11), 999-1013 (2010).

[13] Allport, D. A., [Perspectives on perception and action], ch. Selection for action: Some behavioural and neurophysiological considerations of attention and action, 395-419, Lawrence Erlbaum Associates, Hillsdale, NJ, USA (1987).

[14] Prinzel, L., Freeman, F., Scerbo, M., Mikulka, P., and Pope, A., "A closed-loop system for examining psychophysiological measures for adaptive task allocation," The International Journal of Aviation Psychology 10, 393-410 (2000).

[15] Prinzel, L., Freeman, F., Scerbo, M., Mikulka, P., and Pope, A., "Effects of a psychophysiological system for adaptive automation on performance, workload, and the event-related potential P300 component," Human Factors 45, 601-613 (2003).

[16] Byrne, E. A. and Parasuraman, R., "Psychophysiology and adaptive automation," Biological Psychology 42, 249-268 (1996).

[17] Fragopanagos, N. F. and Taylor, J. G., "Emotion recognition in human-computer interaction," Neural Networks 18(4), 389-405 (2005). 
[18] Yildirim, S., Narayanan, S., and Potamianos, A., "Detecting emotional state of a child in a conversational computer game," Computer Speech \& Language 25, 29-44 (2011).

[19] Daw, N., O’Doherty, J., Dayan, P., Seymour, B., and Dolan, R., "Cortical substrates for exploratory decisions in humans," Nature 441, 876-879 (2006).

[20] Jepma, M. and Nieuwenhuis, S., "Pupil diameter predicts changes in the exploration-exploitation trade-off: Evidence for the adaptive gain theory," Journal of Cognitive Neuroscience 23, 1587-1596 (2011).

[21] Michishita, A., Naito, T., and Shibata, T., "Analysis and modelling of affective japanese sitting postures," in [International Conference on Signal-Image Technology 83 Internet-Based Systems], Yetongnon, K., Dipanda, A., and Chbeir, R., eds., 765-770, IEEE Computer Society, CPS, Kyoto, Japan (2-5 Dec 2013).

[22] Mota, S. and Picard, R. W., "Automated posture analysis for detecting learner's interest level," in [Computer Vision and Pattern Recognition Workshop], 49, IEEE, Madison, Wisconsin, USA (16-22 Jun 2003).

[23] Schrempf, A., Minarik, T., Schossleitner, G., Haller, M., and Gross, S., "PostureCare - towards a novel system for posture monitoring and guidance," in [18th World Congress of the International Federation of Automatic Control (IFAC)], Bittanti, S., Cenedese, A., and Zampieri, S., eds., 18(1), 593-598 (2014).

[24] Ekman, P. and Friesen, W. V., "Head and body cues in the judgment of emotion: A reformulation," Perceptual and Motor Skills 24, 711-724 (1967).

[25] D'Mello, S. K. and Graesser, A. C., "Mining bodily patterns of affective experience during learning," in [3rd International Conference on Educational Data Mining (EDM)], de Baker, R. S. J. and Merceron, A. Jr., P. I. P., eds., 31-40 (June 11-13 2010).

[26] Arnrich, B., Setz, C., La Marca, R., Tröster, G., , and Ehlert, U., "What does your chair know about your stress level?," IEEE Transactions On Information Technology In Biomedicine 14, 207-214 (MAR 2010).

[27] Heyer, P., Herrera-Vega, J., Vila, D.-e., Sucar, L. E., and Orihuela-Espina, F., "Posture based detection of attention in human computer interaction," in [6th Pacific-Rim Symposium on Image and Video Technology. Lecture Notes in Computer Science], Marroquín, J. L. and Tan, T., eds., 220-229 (28th OCT -1st NOV 2013).

[28] Grafsgaard, J. F., Boyer, K. E., Wiebe, E. N., and Lester, J., "Analyzing posture and affect in task-oriented tutoring," in [Proceedings of the Twenty-Fifth International Florida Artificial Intelligence Research Society Conference (FLAIRS)], Youngblood, M. and McCarthy, P. M., eds., 438-443, AAAI Press, Marco Island, Florida, USA (May 2325 2012).

[29] Krakauer, J. W., Carmichael, S. T., Corbett, D., and Wittenberg, G. F., "Getting neurorehabilitation right: what can be learned from animal models?," Neurorehabilitation and Neural Repair 26, 923-931 (OCT 2012).

[30] Kleim, J. A. and Jones, T. A., "Principles of experience-dependent neural plasticity: implications for rehabilitation after brain damage," Journal of Speech, Language, and Hearing Research 51, S225-S239 (FEB 2008).

[31] Holden, M. K., "Virtual environments for motor rehabilitation: Review," CyberPsychology \& Behavior 8(3), 187-211 (2005).

[32] Sucar, L. E., Luis, R., Leder, R., Hernández, J., and Sánchez, I., "Gesture therapy: A vision-based system for upper extremity stroke rehabilitation," in [32nd Annual International Conference of the IEEE Engineering in Medicine and Biology Society (EMBS)], 3690 - 3693, IEEE, Buenos Aires, Argentina (31st AUG -4th SEP 2010).

[33] Mitchell, T. M., [Machine Learning], McGraw-Hill, Inc., New York, NY, USA, 1 ed. (1997).

[34] Martínez-Arroyo, M. and Sucar, L. E., "Learning an optimal naive bayes classifier," in [18th International Conference on Pattern Recognition (ICPR'06)], 3, 1236-1239 (2006).

[35] Escalante, H., Montes, M., and Sucar, L., "Particle swarm model selection," Journal of Machine Learning Research 10, 405-440 (2009).

[36] Bengio, Y., "Learning deep architectures for AI," Foundations and trends in machine learning 2(1), 1-127 (2009). 